\title{
Perspectives on Supervision in Human Services: Gazing Through Critical and Feminist Lenses
}

\author{
Jay Poole \\ The University of North Carolina at Greensboro
}

\begin{abstract}
*Please address all correspondence to: Dr. Jay Poole, MSW, LCSW, Visiting Assistant Professor, Department of Social Work, The University of North Carolina at Greensboro; Email: kjpoole@uncg.edu ; Phone: 336.334 .4097 ; Fax: 336.334.5210.
\end{abstract}

\begin{abstract}
Supervision is a cornerstone activity in the development of practice skills for human service professionals. Traditionally, supervision is conducted in ways that emphasize the expertise of the supervisor and the need to impart expert wisdom to the supervisee. In many ways, the supervisory relationship often mirrors traditional teacher/student roles contextualized in dominant/subordinate ways of being together. Oversight and monitoring become central activities in such traditional supervisory approaches, which may limit possibilities for growth and development. Critical and feminist perspectives offer other ways of conceptualizing supervision, namely through careful consideration of how power operates and may be used in the supervisory relationship. Intentional reciprocity may be discovered if dominant/subordinate power differentials are re-visioned. This article suggests ways that supervision and supervisory relationships may be (re)considered beyond traditional approaches.
\end{abstract}

Key Words: Critical theory, Feminist Theory, Human services, Mentoring, Supervision

F or many years, supervision has been a central tenant of the human services professions (e.g. psychology, counseling, social work), and as professional licensure has become more prominent supervision has been hailed as the space for "gate keeping" with regard to who is or is not granted permission to be a human services professional. Indeed, most licensure boards (e.g., NASW, AAMFT) require supervision as part of the licensure process with the purpose of ensuring that the new licensee is monitored and supported to perform in the best interests of the public. Obviously, the relationship between supervisor and supervisee is one that is important and likely will have a career-long impact on the practice of the supervisee. The intent here is to raise questions about the dynamics that occur in supervisory relationships, with particular focus on the location of power and how that power is understood and addressed in supervision. In this discussion, critical and feminist lenses are used to consider how power in 
supervisory relationships may be re-visioned in a manner that deconstructs some of the traditional dynamics that may be present (e.g. hierarchical structures, dominant/subordinate relationships).

The critical lens employed here involves concepts found primarily in the work of Freire (1998), hooks (2009), and Greene (1988). As Greene (1988) asserts, the critical approach involves making the strange familiar and the familiar strange, calling for (re)examination of what we know and practice through a deconstructive process. Freire (1998) not only supports the notion of careful and meaningful deconstruction of concepts, ideas, and practices that reinforce oppression; he also calls for action to be taken to effect what is believed to be oppressive. Feminist approaches involve calling into question structures and practices that privilege dominant/subordinate binaries, which support hierarchal power relationships grounded in patriarchy. In traditional supervision models, the dynamic of supervisor as dominant and supervisee as subordinate is not only expected, it may be reinforced by practices that reiterate the superiority of knowledge supposedly held by the supervisor (Bogo \& Dill, 2008). If the binary of dominance and subordination can be disrupted, possibilities for more egalitarian and reciprocal supervisory relationships may be imagined.

The meaning of the word supervision, derived from Latin, is grounded in the notion that one is to be overseen or watched (Miriam-Webster Online Dictionary, 2010). In its meaning, supervision implies a hierarchal power structure where one person is deemed to be "in charge" of another in a manner that places responsibility not only on the doer, but also the watcher. Trust is central in this traditional approach to supervision in that an assumption often is made that the doer cannot yet be trusted to do correctly Thus, the watcher is trusted to ensure that the task at hand is completed, usually to a standard set by some higher authority or entity, and/or by the demands of the public and through a means of collaboration with or directives to the supervisee (Kadushin \& Harkness, 2002). Power in the supervisor/supervisee relationship has been addressed in models of curriculum for training supervisors. For example, in their work with child welfare supervisors, Bogo and Dill (2008) point out that there is a lack of knowledge about how power and authority is used in supervision.

It seems important to consider the origins of supervision in human services and as Smith (2005) indicates, early efforts to supervise human service workers may be found in the Charitable Organization Societies in Europe and America in the late $19^{\text {th }}$ century. At the time, those "volunteers/caseworkers" who "visited" the needy often were charged covertly with determining who the deserving poor were. Thus, the need to oversee these decisions became important in terms of ensuring that resources were used efficiently and effectively. In fact, the charge to oversee the distribution of resources exists today particularly in agencies where decisions about scarce resources are made, demanding that workers carefully determine who gets what through an often complex system of evaluation. If we expand this notion of oversight into managed care, we can begin to understand that issues of trust rise again as human service workers are not trusted to deliver services in an efficient and effective manner; thus, the 
management of care (services) becomes central in ensuring efficiency and effectiveness.

No doubt, there have been abuses and misuses of the system of care in human services; yet, have these concerns brought us to a place where human service workers are not believed to be accountable and not to be trusted to deliver services according to particular standards? The National Association of Social Workers (NASW) launched a major effort to address the image of professional social work in 2005 and the literature illustrates that, despite some positive gains in understanding what the profession does, the public continues to struggle with exactly what social work is or is not (Dennison, Poole, \& Qaqish, 2007; LeCroy $\&$ Stinson, 2004). The point here is that the profession of social work, and perhaps other human service disciplines, are not always fully understood or appreciated by the public, which may create a sense of doubt with regard to what social workers and/or human service professionals can and will do. It is possible that doubtfulness, rooted in doubts about the professions themselves, permeates the relationship between supervisor and supervisee, creating an atmosphere of mistrust that must be addressed if effective supervision is to be realized.

If an atmosphere of power sprinkled with doubt and mistrust is present in the supervisor/supervisee relationship, how may it be acknowledged and utilized as a tool in supervision? As Smith (2005) asserts, there are three basic realms in which supervision can and does operate: administrative, educational, and supportive. From the perspective of Smith (2005), effective supervision occurs through the fluidity of work in and among these realms, and when one is able to engage in introspective reflexivity one can enhance internal rapport regarding what one brings to one's professional life. Noble and Irwin (2009) point out that recent economic and socially conservative trends have increased demands for accountability, which has put greater demands on practitioners for productivity and limited the ability to reflect critically on how practice is being conducted. It seems vital to social work practice that reflexive and critical analysis of practice be reclaimed, and I assert supervision is a space where that can occur.

The discussion here facilitated an opportunity for me to reflect on and analyze approaches and practices involved in supervision and the play of power within supervisory relationships. Using reflexivity, a method often associated with feminist research that involves personal reflection and analysis, what follows will include first person references to experience with supervision from the perspective of clinical social work practice. The intent is to offer the reader opportunities to (re)consider how approaches to supervision may be revisited and revised after personal reflective and analytical work has occurred.

\section{Beginning the Supervisory Relationship}

Most of the time, when a new supervisee walks into my office for our first meeting, they bring with them years of culture and socialization that insists that I am an authority and their knowledge largely is irrelevant. Of course, this dynamic is not foreign in many relationships where hierarchy operates as evidenced in the traditional teacher/student relationships or traditional doctor/patient relationships. The reader may note that tradition in such 
relationships refers to contextualization in binarial approaches to being together (e.g. the relationship is based on and supports a dominant/subordinate power differential). Many have asserted that such binaries have origins in patriarchal structures; thus, one of the feminist aspects of this discussion (Butler, 1990; Evans, 1997; Freedman, 2002; Jagose, 1996).

Feminism, taken up as epistemology, offers possibilities for troubling binarial constructs of relationships and I believe that in order to provide meaningful supervision and mentorship in human services, one must trouble the dominant/subordinate binary. This is not to say with a liberal feminist voice that the playing field must be leveled and all power equalized. In fact, I do not believe the power that resides in supervisory relationships can ever be equal; however, it can be acknowledged and used. Additionally, and again taking a feminist position, I believe that power can be a location for productive reflection and introspective work as the supervisor and supervisee disturb notions about constructing and utilizing therapeutic relationships with the people who are or will be served by the supervisee. Of course, a beginning point often reflects the interrogation of what is "normal" with regard to how human services work is done- a point not without controversy.

During the first few sessions of a new supervisory relationship I am forthcoming in discussing locations and operations of power with particular emphasis on the notion that I am some sort of omnipotent paternal figure that will impart wisdom and skill. Of course, I do not discount the fact that years of experience in social work has taught me and others who have spent time in the profession a great deal, and supervisory relationships are a wonderfully rich space to share such wisdom. Yet, if one engages with a supervisee within the binarial frame of knower/knowee without acknowledging what is actually known, unknown, yet to be known, and what one cannot bear to know, one will set a precedent for viewing and being viewed as the authority. For example, I am quick to tell those whom I supervise that I do not have all the answers to their questions and that my view of my role with them is to consider many possibilities, even those which have gone unconsidered in the past.

Authoritarian approaches to supervision, framed in patriarchy, often become problematic in that they may quickly silence what may need to be voiced, particularly with regard to areas that are controversial or that create vulnerability. For example, a person seeking supervision may have some sensitive questions about how to work with particular populations, and because she views her supervisor as an authority (the disciplinarian father/parent), she may want to please the supervisor much like she would a parent; thus, she may not give voice to her concerns and she may or may not seek information from other sources. We are socialized to respect those with authority and the supervisor is certainly in a position of authority. Authoritarianism undermines the creation of safety in a supervisory relationship and without safety those seeking supervision will undoubtedly avoid what feels dangerous. Bogo and Dill (2008) found that when supervisors perceived their supervisees to be competent, authority was shared, particularly with regard to decision-making about cases and other practice actions. However, when competence or trustworthiness was questionable, supervisors 
were much more likely to exercise their power and authority. It is not clear if the supervisors address issues of competence and trust in ways that did not rely on an authoritarian model. Trust seemed to emerge as a central concern in the work Bogo and Dill (2008) did with child welfare supervisors and when trust was eroded, power and authority shifted from being collaborative to being claimed and used by the supervisor. Conversely, relationships that completely avoid any sense of knowing on the part of the supervisor may create a sense of being unsafe.

It has been my experience that those who challenge authority and authoritarian approaches may move to the other end of the spectrum and opt to approach supervision as a location where all power and authority is not only challenged, it is/they are leveled. In this rather unilateral approach, safety is again concerning as the person seeking supervision may perceive that the supervisor does not have any expertise, creating an atmosphere of doubt and mistrust that may arise when a sensitive or vulnerable situation occurs. Thus, finding and creating spaces where power and authority can be acknowledged and used to dispel notions that preclude an open, honest, and trusting relationship is vital to effective and efficient supervision.

I often say to those seeking supervision from me that I do not know all there is to know and that I am constantly learning. I also let them know that I am willing to share what I do know and that working together we can discover multiple ways of viewing situations. I rely on a tenant of critical approaches; namely, unfinishedness, which asserts that we are constantly under construction personally and professionally (Freire, 1998; Greene, 1988). I attempt to create an ongoing atmosphere of learning and discovery as I begin to engage in supervision and I make this as clear as possible to those with whom I am working. For example, a person with whom I recently began supervision wanted to know what he needed to learn, as if I had a prescriptive list that I would give to him. I explained that I had no idea what he needed to learn but that we would certainly explore this question and that we would learn together. A point that I find very important is the idea that I am somehow going to offer a checklist or laundry list of how to approach/handle learning in general or particular situations.

\section{Laundry Lists}

As an educator, I often have students who come into the classroom with the common expectation that I am going to provide them a laundry or checklist of just how to do social work. I find that the laundry list mentality also has a presence with supervisees. I believe that most of us are set up through formal education to expect to be told what to do and when to do it; thus, the expert/learner binary has become reified as "normal" and expected (Purpel \& McLaurin, 2004). The status of "expert" as normal contributes significantly to the power differential in the supervisor/supervisee relationship and must be carefully examined by the supervisor if power is to be critiqued. Further, the emphasis on evidenced-based practice reinforces notions that there are particular ways to engage in social work though there are calls to carefully examine the implications of evidenced-based approaches with regard to prescriptive approaches to human 
services (Barker, 2001; Biesta, 2007; Duncan \& Miller, 2006; Otto, Polutta, \& Ziegler, 2009 ).

Often, I find that those who provide supervision in the role of expert have some sense of ability to control the actions of a supervisee. An example of this approach lies in the supervisor who asserts that his or her "workers" would never make a decision that went against what the supervisee had been told by the supervisor-something I have heard from some supervisors I know. Certainly, a supervisor can influence the actions of the supervisee and it is this function of power that can be useful if viewed through a critical lens. Of course status operates in this realm and is of particular concern when supervision involves administrative oversight. Thus, the supervisor as expert is situated in a space that feels safe as vulnerabilities are kept at bay vis-à-vis protocols and procedures, which so often are important and sometimes useful tools in the laundry list approach to doing social work.

I admit that having laundry lists guidelines often represent a welcome security blanket in tough spots, particularly for someone new to the profession or in situations where supervision may not be readily available at the moment decisions have to be made. There is no doubt that protocols are sometimes the only way to ensure that practice is occurring according to policies and acceptable standards. Be clear that there is no call here to run out and find all agency protocols or Codes of Ethics and shred them; yet, I do assert that discussions about why the policies and protocols exists must be included in supervision, and further, such discussions should be used to critically examine how institutional power is operationalized and how it must be monitored. Van De Luitgaarden (2009) suggests that critical examination of experiential evidence-based practice in social work is important and may yield different perspectives on decisionmaking in social work practice. Further, interrogating the laundry list can serve to develop skills that are needed for complex cases where detail and nuance are vital in assessment, diagnosis, and service planning. The practitioner's ability to engage in thoughtful and competent practice with careful attention to the synergy of theory and practice is of great import and cannot be masked through the ability to simply follow protocol.

The comfort and security that is associated with laundry list practice models can easily become a location for liability and risks.

Building competency in practice must include the careful and critical evaluation of one's practice skills and approaches above and beyond what is deemed as "protocol" or as "clinical pathways." Human dynamics demand that human service providers be well versed in the skills and knowledge that is required to address challenges and to identify strengths. Otto, Polutta, and Ziegler (2009) suggest reflexive professionalism, which is presented as engagement with "...empirically informed yet reflexively contextualized processes through which social workers may appropriately mediate rather than 'effectively prescribe,'..." (p. 477). Indeed, supervision must be the location in which new and seasoned professionals engage in careful and thoughtful evaluation of practice.

I often use the, "What happens if...?" question to trouble the supervisees' comfort level with what has been learned (or not) with regard to prescribed 
assessment and intervention. For example, the person whom I am supervising has determined that a particular intervention is warranted based on the case presentation, evidenced based practice, and our discussion; however, before we end discussion of the case I pose the question, what happens if the client rejects the intervention strategy or does not respond to it in the expected manner? This type of critical questioning helps to speculate on possibilities beyond what have been chosen or prescribed by the evidence. It is important to note that this process also challenges me in critically thinking about what decision-making process is important. Also, I call into question particular protocols even if I believe them to be well grounded in theory and practice in order to engage in critical analysis. I have the person with whom I am working ask me how I would approach particular scenarios and this opens spaces for discussion and critique that enables me to be more reflexive with my own practice. In essence, I attempt to create a reciprocal environment where learning and insight can flourish for me as well as the supervisee.

\section{Reciprocity}

The notion of reciprocity implies that there is give and take or some aspect of sharing between people or entities, and often the idea of sharing in supervision relationships is puzzling, particularly if the relationship is approached traditionally. After all, what does a newcomer to human services have to offer to those of us who have well-seasoned and established careers as professional givers of care? I assert that it is this question that can begin the discussion of how power operates and can be used in the supervisor relationship. Further, if asked seriously, the supervisee can begin to explore what is known and what is being brought to the proverbial table in the supervision relationship.

Turning again to critical thinkers, hooks (2009) offers viewpoints that celebrate the idea that reciprocity fosters an environment rich with opportunities to know and learn in a manner that engages both the supervisor and supervisee in a relationship which relies on mutual respect for what is known and what can be discovered. Plural points-of-view are taken as beneficial to the examination and refinement of ideas and approaches that may or may not be included in protocols or endorsed by particular clinical pathways. Reciprocity seems to disrupt the modernist project of deducing information to pinpoints of so-called truth and (re)introduces the notion that complex human dynamics insist on multiple and often fluid assessments and approaches. Indeed, social justice as a cornerstone of much of social work and other human service work relies on reciprocal approaches and I believe that supervision can and must be a place where principles of social justice are engaged.

I often ask those with whom I am working to begin any discussion with what we each know about the particulars of the situation at hand. For instance, when working through a case, I will not simply ask the supervisee to present the material and his or her analysis while I listen for the shortcomings of his/her approach or analysis as a supervisor acting as expert may do. Rather, I attempt to listen to the details of the situation and I ask questions and interject possible approaches as we go, encouraging the person with whom I am working to ask 
questions and interject what he/she may or may not do with regard to approach/intervention. I often find myself being misled by my experiences with similar cases whereas the person with whom I am working does not often have the disadvantage of pigeonholing particular case dynamics. We mutually discover new approaches or ideas as we use what we know and do not know to reciprocally engage in a critical analysis of the case at hand.

Obviously, the reciprocal approach does not presume that the power dynamic is leveled; rather, it is used in developing an atmosphere of sharing a mutual learning that feels and often is productive for both the supervisor and supervisee. As this environment is nurtured, what I discover is that both of us begin to know ourselves more deeply, which is vital to building competency in clinical practice.

\section{Introspection and Reflexivity}

Perhaps one of the greatest challenges in being a human service professional is engaging in a deep, reflexive critique of one's self in developing an understanding of what one brings to a client/service provider relationship. Austin and Hopkins (2004) point out that development of learning cultures relies on knowledge of one's skills, competencies, biases, beliefs, and values. Indeed, I find that much of my work with supervisees lies in the discovery of what is being brought into the therapeutic relationships that they are forming with their clients. I point out here that through the reciprocal relationship I attempt to establish, I also can learn about my own position as service provider because, as I have asserted, we are constantly under construction.

One aspect of the notion that we are perpetually under construction involves engagement in reflexive processes. Reflexivity implies deconstructive efforts to gain understanding and insight with regard to sociocultural influences and contexts. Feminist approaches typically are contextualized in postmodernism which offers, among other things, a critique of modernism. Of particular interest to human services is the modern-based practice of objectification, particularly related to how we know the world. Traditionally, modernism relies on the idea that we can know in two basic ways, subjectively and objectively. Subjectivity implies some interpretive quality and objectivity implies an absence of interpretation in lieu of unbiased, observable explanations for phenomena. Evans (1997) writes that subjectivity and objectivity do not exist in forms that we have come to understand through traditional lenses; rather, they exist in a mutual relationship because one depends on the other vis-à-vis an interpreter. Arguing "I" and the "World" are strictly correlative. Evans (1997) asserts that it is impossible to maintain that either of these is more real or more basic than the other or that "...one is the mere after-effect of the other..." (p. 16). It is within the interaction of the world and an individual that so-called reality is constructed, and because of the fluidity of this ongoing interaction, reality is constantly being (re)invented. Interestingly, collective realities seem to form as common interpretations take shape and flourish. However, these too are subject to change as new "reality maps," are shaped and formed (Evans, 1997). As I work with supervisees, I make an effort to engage with them in a reflexive process that unpacks their so-called 
reality maps, which in turn assists me with my ongoing development. Often, a touchstone for this process lies in situations that push the proverbial buttons of the person with whom I am working.

A recent supervision session provides an example as the person with whom I am working found himself frustrated by a client situation that involved a person whom he perceived to be self-centered. As we worked through the dynamics of this case, it became apparent that narcissistic issues played a significant role in the reality map of the supervisee. His understanding of this aspect of himself brought a deeper level of knowing to his therapeutic position and he was able to (re)imagine how he could engage with this client in a manner that felt more productive. It is this deep level of reflexive work that offers a location for developing competencies that build upon theoretical frames.

Often, the client/service provider relationship in human services is contextualized traditionally in that bias can be eliminated, or at least set aside. I have heard and have been told by supervisors and educators operating traditionally that one must dismiss one's own interests/feelings when conducting therapy. I believe that this dismissive approach simply serves to silence what must be acknowledged if therapeutic relationships are to be sites for healing and understanding. Feminist epistemologies asserts that understanding one's subjectivity is vital to how and what one can know and it is within this way of knowing that I find opportunities to encourage supervisees and myself to continually engage in reflexive work that reveals positionality and subjectivity relative to the ever-evolving world in which we exist. It is within this introspective work that one may discover a blurry boundary between supervision and counseling.

I often get a question from people with who I am working in supervision about how what we are doing in terms of introspective/reflexive work differs from therapy. I use this opportunity to engage in a discussion about what therapy is, and often, what we discover is that therapy is a process of discovery involving not only insight but solutions that support healing. Supervision, I point out, is also a process of discovery but does not include personal healing as its goal. Rather, supervision holds its focus on deep understanding in order to better serve and support clients and client systems. If healing needs to occur, I encourage those with whom I am working to seek therapy as a space for healing work.

An example may be found in a case where one of my supervisees discovered that she held particular beliefs about patriarchal/fraternal figures, which was interfering with her ability to empathize with clients who were dealing with patriarchal/fraternal dynamics. Her introspective effort was beneficial to her understanding of what she brought to her own clinical work; yet, the supervisory space was not appropriate for working toward healing. I encouraged her to seek therapy for this concern and we continued to talk about how it played out in her clinical work.

\section{Conclusions}

It is important to say that perhaps the most obvious conclusion here is that there are no conclusions. Rather, what is offered are thoughts that may be taken 
up as one considers supervision and the dynamics in supervisory relationships. Supervisory work is not prescriptive and demands careful and thoughtful planning and engagement. Critical perspectives that incorporate feminist epistemologies in the examination and use of power in supervisory relationships are encouraged. Reciprocal and introspective work is vital to the development of competencies that are important in human services with careful attention to subjective positions related to values that are held as important; self determination, respect for differences, dignity, worth, and social justice. The role of the supervisor is not to function as an omnipotent authoritarian; rather, the supervisor must act reciprocally and deliberately as teacher/learner/facilitator working in tandem with the person whom is seeking supervision. Above all, the well being of those who are and will be served is central in supervisory relationships and power within those relationships must be used toward and for the betterment of how and what one does as a human service professional.

\section{References}

Austin, M. \& Hopkins, K. (2004). Supervision as collaboration in the human services: Building a learning culture. Thousand Oaks, CA: Sage Publications.

Barker, P. (2001). The ripples of knowledge and the boundaries of practice: The problem of evidence in psychotherapy research. International Journal of Psychotherapy, 6, 11-24.

Biesta, G. (2007). Why "what works" won't work: Evidence-based practice and the democratic deficit in educational research. Education Theory, 57, 1-22.

Bogo, M., \& Dill, K. (2008). Walking the tightrope: Using power and authority in child welfare supervision. Child Welfare, 87(6), 141-57.

Butler, J. (1990). Gender trouble: Feminism and the subversion of identity. New York: Routledge.

Dennison, S., Poole, J., \& Qaqish, B. (2007). Students' perceptions of social work: Implications for strengthening the image of social work among college students. Social Work, 52 (4), 350-360.

Duncan, B., \& Miller, S. (2006). Treatment manuals do not improve outcomes. In J. Norcross, R. Levant, \& L. Beutler. (Eds.), Evidence-based practices in mental health: Debate and dialogue on the fundamental questions (pp. 140-149). Washington, DC: American Psychological Association.

Evans, A. (1997). Critique of patriarchal reason. San Francisco: White Crane Press. 
Freedman, E. (2002). No turning back: The history of feminism and the future of women. New York: Ballantine Books.

Freire, P. (1998). Pedagogy of freedom: Ethics, democracy and civic courage. New York: Rowman \& Littlefield Publishers, Inc.

Greene, M. (1988). The dialectic of freedom. New York: Teachers College Press.

hooks, B. (2009) Teaching critical thinking: Practical wisdom. New York: Routledge.

Jagose, A. (1996). Queer theory: An introduction. New York: University of New York Press.

Kadushin, A., \& Harkness, D. (2002). Supervision in social work (4th ed.). New York: Columbia University Press.

LeCroy, C. \& Stinson, E. (2004). The public's perception of social work: Is it what we think it is? Social Work, 49, 164-174.

Noble, C. \& Irwin, J. (2009). Social work supervision: An exploration of the current challenges in a rapidly changing social, economic, and political environment. Journal of Social Work, 9 (3), 345-358.

Otto, H., Polutta, A., \& Ziegler, H. (2009). Reflexive professionalism as a second generation of evidence-based practice: Some considerations on the special issue "What works? Modernizing the knowledge-base of social work". Research on Social Work Practice, 19(4), 472.

Purpel, D. \& McLaurin, W. (2004). Reflections on the moral and spiritual crisis in education. Washington, D.C.: Peter Lang.

Smith, M. K. (1996, 2005). The functions of supervision, the encyclopedia of informal education. Retrieved on October 1, 2009 from http://www.infed.org/biblio/functions_of_supervision.htm last update: September 03, 2009. supervision. (2010). In Merriam-Webster Online Dictionary. Retrieved March 29, 2010, from http://www.merriamwebster.com/dictionary/supervision

Van De Luitgaarden, G. (2009). Evidence-based practice in social work: Lessons from judgment and decision-making theory. British Journal of Social Work, 39(2), 243-260. 